\title{
Avaliação dos níveis séricos de hormônios tireoidianos em araras (Ara spp.) pelo método de quimioluminescência
}

[Evaluation of serum levels of thyroid hormones in macaws (Ara spp.) by chemiluminescence]

\author{
K.R.A. Vieira1, Z.S. Cubas², W. de Moraes², M. Dislich³, L.R. Oliva , A.R. Lobo Júnior ${ }^{4}$, \\ M.I.S. Santana ${ }^{1}$ \\ ${ }^{1}$ Universidade de Brasília - UnB - Brasília, DF \\ ${ }^{2}$ Itaipu Binacional - Foz do Iguaçu, PR \\ ${ }^{3}$ Parque das Aves Foz Tropicana - Foz do Iguaçu, PR \\ ${ }^{4}$ Universidade Federal dos Vales do Jequitinhonha e Mucuri - UFVJM - Unaí, MG
}

\section{RESUMO}

Este trabalho investigou a eficiência do método de quimioluminescência para obtenção das concentrações séricas de hormônios tireoidianos em 23 exemplares de araras [Ara ararauna $(\mathrm{n}=12)$ e Ara chloropterus $(\mathrm{n}=11)]$, de ambos os sexos [machos $(\mathrm{n}=11)$ e fêmeas $(\mathrm{n}=12)]$, mantidas em cativeiro. Os valores de T4 total, T4 livre, T3 livre e TSH variaram, respectivamente, de 0,3 a $2,5 \mu \mathrm{g} / \mathrm{dL}$ (média=0,94 $\mu \mathrm{g} / \mathrm{dL}$ ), 0,11 a $0,46 \eta \mathrm{g} / \mathrm{dL}$ (média=0,28ng/dL), 1,46 a $3,59 \mathrm{pg} / \mathrm{mL}($ média $=2,42 \mathrm{pg} / \mathrm{mL}$ ) e 0,01 a $0,12 \mu \mathrm{UI} / \mathrm{mL}$ (média=0,04 $\mu \mathrm{UI} / \mathrm{mL}$ ). Houve interação entre espécie e sexo $(\mathrm{P}<0,05)$ sobre as concentrações de $\mathrm{T} 4$ total: os machos possuem maiores valores do que as fêmeas de Ara ararauna, enquanto as fêmeas de Ara chloropterus dispõem de níveis mais altos do que os machos desta espécie. Efeito de sexo não foi observado (P>0,05) para os valores de T4 livre, T3 livre e TSH, mas o efeito de espécie foi constatado $(\mathrm{P}<0,05)$ para as concentrações de T3 livre. Neste caso, as concentrações de T3 livre são menores em Ara ararauna do que em Ara chloropterus. Os intervalos de referência obtidos sugerem que é possível mensurar as concentrações séricas de hormônios tireoidianos em araras pelo método de quimioluminescência, devendo-se considerar variações relevantes entre diferentes espécies e sexos.

Palavras-chave: aves, hormônios da tireoide, imunoquimioluminescência

\begin{abstract}
This paper investigated the efficiency of chemiluminescence in obtaining serum concentrations of thyroid hormones in twenty-three macaws kept in captivity, from two sexes [male $(n=10)$ and female $(n=12)$ ] and two species [Ara ararauna $(n=12)$ and Ara chloropterus $(n=11)$ ]. Reference intervals for the hormones sampled were indicated and the effects of species and sex on these values were analyzed. Concentration values of total T4, free T4, free T3 and TSH varied respectively from 0,3 to 2,5 $\mu \mathrm{g} / \mathrm{dL}$ (average $=0,94 \mu \mathrm{g} / \mathrm{dL}$ ), $0,11-0,46 \eta \mathrm{g} / \mathrm{dL}$ (average $=0,28 \eta g / d L), 3,59-1,46 \mathrm{pg} / \mathrm{mL}$ (average $=2,42 \mathrm{pg} / \mathrm{mL}$ ), and $0,01-0,12 \mu U I / \mathrm{mL}$ (average $=$ $0,04 \mu U \mathrm{I} / \mathrm{mL})$. There was interaction between species and sex $(P<0.05)$ on total T4 concentrations. Males had higher values than females in Ara ararauna, while females had higher values than males in Ara chloropterus. Sex effect was not observed (P>0.05) for concentrations of free T4, free T3 and TSH, but species effect was observed $(P<0.05)$ for free T3 concentrations. In this case, concentrations of free T3 were lower in Ara ararauna than in Ara chloropterus. The results of this study suggest that it is possible to measure serum concentration of thyroid hormones in macaws by means of chemiluminescence method, as long as relevant variations between different species and sexes are taken into account.
\end{abstract}

Keywords: birds, thyroid hormones, thyroxine, immunochemiluminescence

\section{INTRODUÇÃO}

Entre o grupo de doenças clínicas relacionadas à glândula tireoide já documentadas em aves, que inclui o bócio, o hipo e o hipertireoidismo, a tireoidite autoimune e as neoplasias, o bócio hiperplásico é a doença tireoidiana mais relatada (Orosz et al., 2016). Evidências indicam que os psitacídeos são mais suscetíveis e possuem maior prevalência de bócio do que outras famílias de

Recebido em 12 de dezembro de 2016

Aceito em 15 de março de 2017

E-mail: kassia.rav@gmail.com 
aves (Loukopoulos et al., 2014). Oglesbee (1992) confirmou um caso de hipotireoidismo em araracanga (Ara macao), bem como há casos de suspeita clínica reportados em outras espécies de psitacídeos (Merryman e Buckles, 1998).

O diagnóstico laboratorial e a monitoração de doenças da tireoide são baseados na determinação das concentrações séricas dos hormônios tireoidianos (Shamsian et al., 2016). Vários métodos têm sido empregados para mensurar essas concentrações no soro, e o radioimunoensaio (RIA) é o mais utilizado, por sua acurácia e sensibilidade, mas requer tempo de incubação prolongado, licença dos órgãos normatizadores para o uso de isótopos radioativos, além de dispositivos de segurança e eliminação específica de resíduos, pois oferece perigo de radioatividade para os operadores, condições que levaram ao desenvolvimento de métodos alternativos (Eshratkhah et al., 2011; Shamsian et al., 2016).

Um dos métodos alternativos é a quimioluminescência (CLIA), cada vez mais utilizada, por ser prática, rápida, barata e sem gerar resíduos radioativos e nenhum efeito tóxico dos reagentes (Lemos et al, 2015; Shamsian et al., 2016). Esse teste foi validado com boa sensibilidade para a dosagem de hormônios tireoidianos em cães, gatos e equinos, de modo que se enquadra como um potencial substituto para o radioimunoensaio (Singh et al., 1997).

O objetivo deste trabalho foi investigar a eficiência do método de quimioluminescência na dosagem de níveis séricos de hormônios tireoidianos em araras das espécies Ara ararauna e Ara chloropterus. Obtiveram-se os valores médios e limites inferiores e superiores para os hormônios tireoidianos analisados. Por meio de métodos estatísticos, avaliaram-se as variações entre animais de espécie e sexo diferentes. A técnica de quimioluminescência foi proposta como uma alternativa eficiente, simples, barata, rápida e segura de diagnóstico de doenças da tireoide nas espécies estudadas.

\section{MATERIAL E MÉTODOS}

Este projeto foi aprovado pelo Comitê de Ética no Uso Animal da Universidade de Brasília (protocolo $\left.\mathrm{n}^{\circ} 66699 / 2016\right)$ ). O presente trabalho utiliza 23 exemplares de araras de duas diferentes espécies: 12 Ara ararauna (araracanindé), quatro machos e oito fêmeas, e 11 Ara chloropterus (arara-vermelha), sete machos e quatro fêmeas. As araras, previamente sexadas pela técnica de biologia molecular, são de diferentes idades, clinicamente saudáveis (com base em exames físico, hematológico e de bioquímica sérica) e estão mantidas no Criadouro de Animais Silvestres da Itaipu Binacional e no Parque das Aves Foz Tropicana, ambos localizados em Foz do Iguaçu, Paraná. Todas as aves vivem em recintos coletivos, recebendo água ad libitum e dieta composta de ração comercial própria para araras e frutas frescas, verduras e legumes diversos nas duas instituições.

Após a captura individual em seu recinto com auxílio de puçá, conteve-se fisicamente cada arara para os procedimentos de identificação, pesagem, exame físico e coleta de sangue. Coletaram-se $3 \mathrm{~mL}$ de sangue da veia jugular direita, no horário das 10 às 12 horas, nos meses de fevereiro e março de 2016. O sangue coletado foi dividido em tubo com anticoagulante (heparina) e tubo com gel ativador de coagulação. Por meio da coleta em tubos contendo heparina, o Laboratório Ambiental da Itaipu Binacional realizou o hemograma completo e os testes de bioquímica sérica.

Em até 30 minutos após a coleta, centrifugaramse os tubos com ativador de coágulo a 6000rpm por 10 minutos para a obtenção de $0,5 \mathrm{~mL}$ de soro. As amostras de soro foram enviadas em isopor com gelo, via transporte aéreo, ao Laboratório Grupo São Camilo (Maringá, Paraná) para a dosagem sérica de hormônios tireoidianos. O laboratório citado realizou as dosagens de tri-iodotironina livre (T3 livre) em $\mathrm{pg} / \mathrm{mL}$, tiroxina total ( $\mathrm{T} 4$ total) em $\mu \mathrm{g} / \mathrm{dL}$, tiroxina livre (T4 livre) em ng/dL, e o hormônio estimulador da tireoide (TSH) em $\mu \mathrm{UI} / \mathrm{mL}$, pelo método de quimioluminescência direta, com o equipamento automático ADVIA Centaur® XP SIEMENS. Os kits são imunoensaios competitivos, largamente empregados em humanos, executados de acordo com as instruções do fabricante (ADVIA Centaur®, Siemens Healthcare Diagnostics, USA).

Realizou-se uma análise de variância para todas as variáveis segundo um delineamento inteiramente ao acaso, em um arranjo fatorial 2 
(espécie) $\times 2$ (sexo), mediante o uso de um modelo linear misto que contempla os efeitos fixos de espécie, sexo e suas interações e o efeito aleatório de origem. A idade e o peso das araras estão incluídos como covariáveis. Na condução das análises de variância dos dados, utilizou-se o procedimento MIXED, do software Statistical Analysis Systems, versão 9.2 (SAS Institute, USA). Para considerar um efeito significante, adotou-se um nível de probabilidade de $5 \%$ ou menor para o teste $F$. Estão apresentados nos resultados a média e o erro-padrão para todas variáveis em função dos tratamentos.

\section{RESULTADOS E DISCUSSÃO}

As aves amostradas estão clinicamente saudáveis e alertas, com escore corporal normal e postura em estação. Os pesos médios das fêmeas $(n=8)$ e machos $(n=4)$ das araras-canindés são de 1,03 $(0,03)$ e $1,1(0,04) \mathrm{kg}$, respectivamente, enquanto os pesos médios das fêmeas $(n=4)$ e machos $(\mathrm{n}=7)$ das araras-vermelhas são de 1,22 $(0,25)$ e $1,26(0,17) \mathrm{kg}$. As médias de peso corporal obtidas estão de acordo com os valores esperados para essas espécies (Carpenter, 2010).

Os resultados dos parâmetros hematológicos (Tab. 1) e dos testes bioquímicos (Tab. 2) estão de acordo com os valores normais para as espécies amostradas (Teare, 2002). Grande parte dos dados clínicos disponíveis para aves é originária de estudos realizados em outros países, onde o clima e o manejo divergem, na maioria das vezes, daqueles encontrados no Brasil, o que pode influenciar nas avaliações laboratoriais (Valle et al., 2008). Dessa forma, os resultados obtidos para a amostra em questão visam colaborar na geração de valores hematológicos e bioquímicos de referência para a região do Brasil onde vivem os animais amostrados.

Tabela 1. Valores hematológicos médios e erro-padrão em araras (Ara ararauna e Ara chloropterus), mantidas no Criadouro de Animais Silvestres da Itaipu Binacional e no Parque das Aves Foz Tropicana, ambos localizados em Foz do Iguaçu, Paraná

\begin{tabular}{|c|c|c|c|c|}
\hline Variáveis & Ara spp. & Ara ararauna & Ara chloropterus & Valores de referência* \\
\hline $\mathrm{N}$ & 23 & 12 & 11 & valores de rererencia* \\
\hline RBC (milhões/mm) & $2,8(0,06)$ & $2,8(0,1)$ & $2,9(0,06)$ & 2,11 a 6,54 \\
\hline $\mathrm{Hg}(\mathrm{g} / \mathrm{dL})$ & $15,1(0,2)$ & $15,5(0,3)$ & $14,8(0,3)$ & 6,2 a 18 \\
\hline VGM (fL) & $158,2(3,7)$ & $163,5(5,2)$ & $152,5(5,0)$ & 55,9 a 229 \\
\hline HGM (pg) & $52,8(1,7)$ & $56,2(1,8)$ & $49,2(2,6)$ & 13,4 a 64,3 \\
\hline $\mathrm{CHCM}(\%)$ & $33,5(0,5)$ & $34,4(0,4)$ & $32,6(1,0)$ & 13,6 a 43,1 \\
\hline $\mathrm{HT}(\%)$ & $44,4(0,7)$ & $45,0(1,0)$ & $43,6(0,9)$ & 34 a 62 \\
\hline $\mathrm{WBC}\left(\mathrm{x} 10^{3} / \mathrm{mm}^{3}\right)$ & $12,1(1,2)$ & $10,1(1,3)$ & $14,5(2,0)$ & 3 a 54,5 \\
\hline $\operatorname{EOS}(\%)$ & $0,01(0,01)$ & $0,0(0,0)$ & $0,02(0,01)$ & 0,0 a 1,3 \\
\hline BAS $(\%)$ & $0,28(0,1)$ & $0,22(0,03)$ & $0,34(0,1)$ & 0,0 a 1,2 \\
\hline MON (\%) & $0,63(0,2)$ & $0,43(0,1)$ & $0,85(0,2)$ & 0,0 a 2,5 \\
\hline $\operatorname{LIN}(\%)$ & $4,2(0,4)$ & $3,6(0,4)$ & $4,88(0,5)$ & 0,3 a 25,7 \\
\hline $\operatorname{HET}(\%)$ & $9,3(1,4)$ & $6,7(1,0)$ & $12,2(1,6)$ & 0,9 a 50,7 \\
\hline $\mathrm{PP}(\mathrm{g} / \mathrm{dL})$ & $3,5(0,13)$ & $3,5(0,24)$ & $3,6(0,13)$ & 1,9 a 7,7 \\
\hline
\end{tabular}

*Fonte: Teare (2002). RBC= contagem total de hemácias; Hg= hemoglobina; VGM= volume globular médio; HGM= hemoglobina globular média; $\mathrm{CHCM}=$ concentração de hemoglobina globular média; HT= hematócrito; WBC= leucócitos totais; $\mathrm{EOS}=$ eosinófilos; $\mathrm{BAS}=$ basófilos; $\mathrm{MON}=$ monócitos; $\mathrm{LIN}=$ linfócitos; $\mathrm{HET}=$ heterófilos; $\mathrm{PP}=$ proteína plasmática; $\mathrm{N}=$ número de indivíduos.

Tabela 2. Média e erro-padrão das variáveis de bioquímica sérica em araras (Ara ararauna e Ara chloropterus), mantidas no Criadouro de Animais Silvestres da Itaipu Binacional e no Parque das Aves Foz Tropicana, localizados ambos em Foz do Iguaçu, Paraná

\begin{tabular}{clccc} 
Variáveis & Ara spp. & Ara ararauna & Ara chloropterus & \multirow{2}{*}{ Valores de referência* } \\
\cline { 1 - 3 } $\mathrm{N}$ & 16 & 10 & 6 & \\
\hline AST (mg/dL) & $155(15,5)$ & $155,2(25,2)$ & $154,3(5,6)$ & \\
Ácido úrico (mg/dL) & $6,5(0,8)$ & $7,7(1,1)$ & $4,5(0,7)$ & 0 a 528 \\
\end{tabular}

*Fonte: Teare (2002). AST = aspartatoaminotransferase; $\mathrm{N}=$ número de indivíduos. 
Na Tab. 3, estão a média e o erro-padrão dos hormônios tireoidianos dosados, conforme sexo e espécie. Houve diferença significativa $(\mathrm{P}<0,05)$ nos níveis de T4 total entre aves de espécie e sexo diferentes (Fig. 1). Os níveis de T3 livre são significativamente menores $(\mathrm{P}<0,05)$ em Ara ararauna do que em Ara chloropterus.

O nível de T4 total variou de 0,3 a $2,5 \mu \mathrm{g} / \mathrm{dL}$, com uma média de $0,94 \mu \mathrm{g} / \mathrm{dL}$. Eshratkhah et al. (2011) mensuraram, por quimioluminescência, as concentrações séricas de hormônios tireoidianos em frangos de corte e obtiveram uma concentração média para T4 total semelhante à apresentada neste trabalho. A média de T4 total em Ara ararauna, determinada por RIA ultrassensível, variou de 0,16 a $0,38 \mu \mathrm{g} / \mathrm{dL}$, com uma média de 0,26 (Greenacree et al., 2001), valores relativamente mais baixos aos encontrados no presente estudo.

Sabe-se que existem variações nos intervalos de referência entre as espécies e, em alguns casos, entre aves da mesma espécie (Orosz et al., 2016). Este trabalho obteve valores de T3 livre e T4 total significativamente maiores em Ara chloropterus do que em Ara ararauna (Tab. 3). Generalizações sobre as diferenças nos níveis de hormônios tireoidianos entre espécies não são possíveis, visto que vários fatores influenciam o funcionamento da tireoide, como a disponibilidade de alimentos e de iodo na dieta, a composição de alimentos, a sazonalidade, a temperatura, a idade, a maturidade sexual, a data da coleta de sangue e o estresse (Greenacre et al., 2001; Schmidt e Reavill, 2008). Portanto, os valores do intervalo de referência devem ser utilizados com cuidado (Orosz et al., 2016), devendo-se comparar somente valores gerados pelo mesmo método de análise e, se possível, do mesmo laboratório e entre aves da mesma espécie. Os resultados devem ser interpretados juntamente com outros indicadores, como sinais clínicos e outros testes laboratoriais (Schmidt e Reavill, 2008).

Tabela 3. Efeito de espécie e/ou sexo sobre os níveis séricos de hormônios tireoidianos em araras (Ara ararauna e Ara chloropterus), mantidas no Criadouro de Animais Silvestres da Itaipu Binacional e no Parque das Aves Foz Tropicana, ambos localizados em Foz do Iguaçu, Paraná

\begin{tabular}{|c|c|c|c|c|c|c|c|c|}
\hline \multirow{3}{*}{ Variável } & \multicolumn{4}{|c|}{ Espécie $(\mathrm{E})$} & \multirow{3}{*}{$\begin{array}{c}\text { Sexo }(\mathrm{S}) \\
\text { Fêmea } \\
(\mathrm{n}=12)\end{array}$} & \multicolumn{3}{|c|}{ Valor de $P$} \\
\hline & Ara spp. & Ara ararauna & Ara chloropterus & Macho & & \multirow{2}{*}{$\mathrm{E}$} & \multirow{2}{*}{$\mathrm{S}$} & \multirow{2}{*}{$\mathrm{E} \times \mathrm{S}$} \\
\hline & $(n=23)$ & $(n=12)$ & $(\mathrm{n}=11)$ & $(n=11)$ & & & & \\
\hline $\begin{array}{l}\text { T3 livre } \\
\text { (pg/mL) }\end{array}$ & $\begin{array}{c}2,42 \\
(0,56)\end{array}$ & $\begin{array}{c}2,1 \\
(0,23)^{b}\end{array}$ & $\begin{array}{c}2,9 \\
(0,23)^{\mathrm{a}}\end{array}$ & $\begin{array}{c}2,4 \\
(0,22)\end{array}$ & $\begin{array}{c}2,6 \\
(0,21)\end{array}$ & 0,02 & 0,52 & 0,72 \\
\hline $\begin{array}{c}\text { T4 total } \\
(\mu \mathrm{g} / \mathrm{dL})\end{array}$ & $\begin{array}{c}0,94 \\
(0,56)\end{array}$ & $\begin{array}{c}0,5 \\
(0,14)^{b}\end{array}$ & $\begin{array}{c}1,6 \\
(0,14)^{\mathrm{a}}\end{array}$ & $\begin{array}{c}0,8 \\
(0,13)^{\mathrm{B}}\end{array}$ & $\begin{array}{c}1,2 \\
(0,13)^{\mathrm{A}}\end{array}$ & $<0,01$ & 0,02 & $<0,01$ \\
\hline $\begin{array}{l}\text { T4 livre } \\
\text { (ng/dL) }\end{array}$ & $\begin{array}{c}0,28 \\
(0,05)\end{array}$ & $\begin{array}{c}0,30 \\
(0,028)\end{array}$ & $\begin{array}{c}0,25 \\
(0,030)\end{array}$ & $\begin{array}{c}0,27 \\
(0,025)\end{array}$ & $\begin{array}{c}0,28 \\
(0,026)\end{array}$ & 0,34 & 0,87 & 0,45 \\
\hline $\begin{array}{c}\text { TSH } \\
(\mu \mathrm{UI} / \mathrm{mL})\end{array}$ & $\begin{array}{c}0,04 \\
(0,04)\end{array}$ & $\begin{array}{c}0,02 \\
(0,015)\end{array}$ & $\begin{array}{c}0,06 \\
(0,016) \\
\end{array}$ & $\begin{array}{c}0,04 \\
(0,014) \\
\end{array}$ & $\begin{array}{c}0,04 \\
(0,014) \\
\end{array}$ & 0,21 & 0,84 & 0,13 \\
\hline
\end{tabular}

Média de quadrados mínimos (erro-padrão); $n=$ número de animais. ${ }^{£}$ Variável influenciada significativamente pela interação de espécie e sexo, a qual foi desdobrada na análise de variância para ambos os fatores na figura abaixo (Fig. 1). ${ }^{\text {a,b }}$ Médias de quadrados mínimos seguidas por diferentes letras minúsculas entre as espécies diferem estatisticamente a um nível de significância de $5 \%$ pelo teste $F$. ${ }^{\mathrm{A}, \mathrm{B}}$ Médias de quadrados mínimos seguidas por diferentes letras maiúsculas entre os sexos diferem estatisticamente a um nível de significância de 5\% pelo teste $F$.

Visto que a proteína carreadora denominada globulina ligadora de tiroxina (TBG) está ausente nas aves, os níveis de T4 livre são menos afetados pelas concentrações de proteína e doenças concomitantes, e sua concentração parece ter maior nível em amostras de aves quando comparadas às de mamíferos. Com isso, o uso de T4 livre para auxílio no diagnóstico de doenças da tireoide em aves tem sido proposto e investigado (Orosz et al., 2016). O intervalo normal de T4 livre para papagaio-da-hispaniola pelo método da diálise de equilíbrio foi de 0,046 a 0,54ng/dL (Brandão et al., 2014), semelhante aos valores obtidos para as espécies de araras no presente estudo, cujos valores variaram de 0,11 a $0,46 n g / d L$.

Embora as concentrações de T4 sejam responsáveis pela geração de várias respostas hormônio-dependentes em aves, é observado que 
T3 é o hormônio fisiologicamente mais ativo (Welcker et al., 2013). Essa afirmação é baseada nas seguintes evidências: i) os receptores tireoidianos em aves, assim como nos mamíferos, sugerem uma maior afinidade para $\mathrm{T} 3$; ii) a proporção relativa entre T3/T4 é maior em aves do que em mamíferos, o que sugere uma boa capacidade de ligação de T3; e iii) as características e o funcionamento das enzimas responsáveis pela produção dos hormônios tireoidianos demonstram maior resposta fisiológica das aves ao T3 (Mcnabb, 2000). Devido à importância fisiológica do T3 em aves, foi realizada a dosagem desse hormônio em todas as araras amostradas, obtendo-se valores em um intervalo de 1,46 a $3,59 \mathrm{pg} / \mathrm{mL}$.

$\mathrm{Na}$ maioria dos intervalos de referência publicados para T4 total em aves, utilizou-se um kit de RIA desenvolvido para sorologia em cães (Greenacre et al., 2001; Orosz et al., 2016) e ainda não validado para o uso nessa classe. Aves apresentam níveis séricos de hormônios da tireoide muito baixos (Mayer e Donelly, 2013), portanto é necessário escolher um teste que detecte com precisão concentrações relativamente baixas. A concentração mais baixa disponível para o kit de RIA é de $0,5 \mu \mathrm{g} / \mathrm{dL}$ (Greenacre, 2009), enquanto a sensibilidade analítica mínima do kit de CLIA utilizado neste trabalho é de $0,3 \mu \mathrm{g} / \mathrm{dL}$. Considerando-se esses fatos, sugere-se a realização de novos estudos que comparem os valores dos hormônios tireoidianos de araras obtidos pelos métodos de RIA e CLIA, com amostras dos mesmos animais e analisadas em um mesmo laboratório, com o objetivo de se definir qual ensaio é mais preciso e de se criarem intervalos de referência confiáveis nas espécies de araras estudadas.

Os níveis de TSH neste trabalho variaram de 0,01 a $0,12 \mu \mathrm{UI} / \mathrm{mL}$, com uma média de $0,04 \mu \mathrm{UI} / \mathrm{mL}$. Entre os 23 animais, 13 obtiveram concentração de TSH próxima ao valor mínimo detectável pelo kit $(0,008 \mu \mathrm{UI} / \mathrm{mL})$. Esses resultados podem indicar que os níveis de TSH nessas espécies são naturalmente baixos. Outra explicação possível para esses valores baixos está associada ao fato de que, em aves adultas, os valores circulantes de hormônios da tireoide flutuam em ciclos anuais (Groscoulas e Leloup, 1986; Silverin et al., 1989) devido à interação sinergética com outros hormônios, especialmente no final da temporada reprodutiva e durante a muda (Norris e Carr, 2013). Assim, o fato de as aves deste estudo estarem fora do período reprodutivo e a amostragem ter sido realizada em um curto período de tempo podem ter influenciado nos valores de TSH. Recomenda-se a realização de novas pesquisas que incluam dosagens dos hormônios tireoidianos em todos os períodos do ano, com o objetivo de avaliar a influência da sazonalidade nos níveis de hormônios tireoidianos nessas espécies.

Os resultados das dosagens hormonais sugerem valores fisiológicos de referência para hormônios tireoidianos, pelo método de quimioluminescência, para Ara ararauna e Ara chloropterus. A obtenção desses valores tem grande importância no diagnóstico de alterações na função tireoidiana em aves enfermas. Com base em pesquisas diagnósticas bem embasadas, o papel que a glândula tireoide desempenha em doenças clínicas em aves será mais compreendido. A quimioluminescência é um teste prático, barato e rápido, que não gera resíduos radioativos, nenhum efeito tóxico dos reagentes (Shamsian et al., 2016; Lemos et al., 2015) e torna acessível a realização de testes tireoidianos na rotina clínica.

A Fig. 1 apresenta as diferenças na concentração de T4 total, conforme a espécie e o sexo das aves. Os machos têm valores maiores $(\mathrm{P}<0,05)$ de T4 que as fêmeas na espécie Ara ararauna, enquanto as fêmeas possuem valores maiores $(\mathrm{P}<0,05)$ que os machos na espécie Ara chloropterus.

Embora não completamente elucidados, fatores reprodutivos podem ser responsáveis por variações nas concentrações dos hormônios tireoidianos (Johnson, 1994). Diferenças entre sexos nas concentrações de tiroxina total são reportadas em ratos e cobaias, e observou-se que machos apresentam valor de T4 sérico maior que fêmeas (Segal et al., 1982; Muller et al., 2009). Em gatos, estudos avaliaram a influência do sexo sobre os valores de T4 total, mas os resultados são conflitantes e sem consenso (Vieira et al., 2010). Em aves, essa relação não está esclarecida. Em vista disso, necessita-se de mais estudos que testem a representação de subgrupos, como sexo e idade, nas variações dos níveis séricos desses hormônios, com o objetivo de se desenvolverem resultados precisos. 


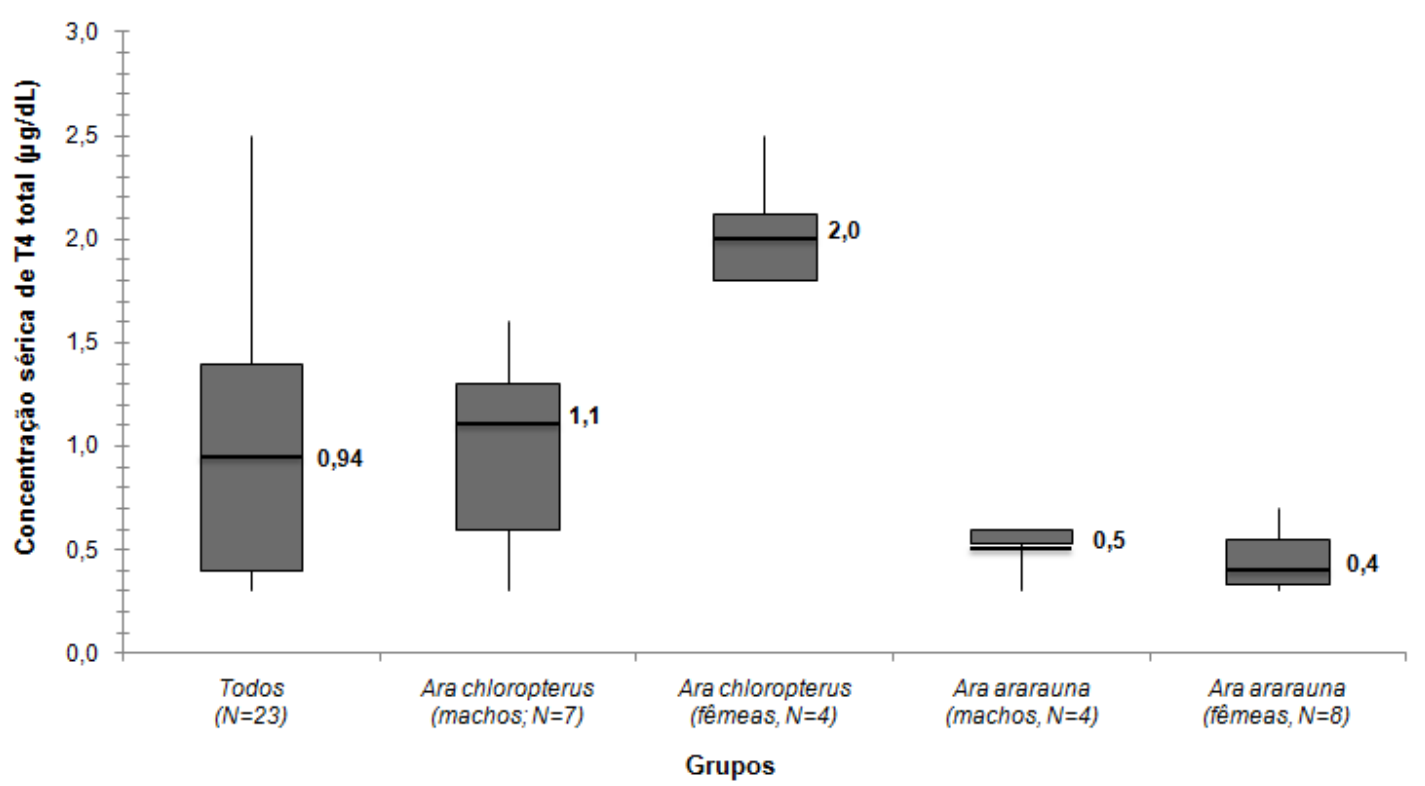

Figura 1. Gráfico de caixa demonstrando a interação entre espécie e sexo sobre a concentração total do hormônio tiroxina ( $\mathrm{T} 4$ total, $\mu \mathrm{g} / \mathrm{dL}$ ) em araras. Os valores dentro do gráfico indicam as médias de quadrados mínimos. $\mathrm{N}=$ número de indivíduos para as combinações.

\section{CONCLUSÕES}

O método de quimioluminescência se mostrou eficiente para a obtenção das concentrações séricas de hormônios tireoidianos em araras e apresenta os valores fisiológicos para esses hormônios em Ara ararauna e Ara chloropterus. As concentrações dos hormônios tireoidianos somente podem ser comparadas com os valores de referência estabelecidos na atual pesquisa, quando se utilizar o mesmo método de análise e, se possível, o mesmo laboratório, além de considerar as variações relevantes entre diferentes espécies e sexos. As dosagens de hormônios tireoidianos devem fazer parte da rotina clínica de aves, pois são variáveis fundamentais para um diagnóstico preciso.

\section{REFERÊNCIAS}

BRANDÃO, J.; RICK, M.; BEAUFRERE, H. et al. Free and total thyroid hormones reference interval in the Hispaniolan Amazon parrot. Proc Assoc Avian Vet Annu. Conf., v.29, 2014.

CARPENTER, J.W. Formulário de animais exóticos. 3.ed. São Paulo: MedVet, 2010. 578p.

Arq. Bras. Med. Vet. Zootec., v.70, n.1, p.174-180, 2018
ESHRATKHAH, B.; ZADEH, S.A.; FOROUZAN, $\mathrm{V}$. et al. Comparative study on the determination of serum thyroid hormones by two methods of immunoassay in broiler breeder poultry. Comp. Clin. Pathol., v.20, p.337-340, 2011.

GREENACRE, C.B. Diagnostic testing in the field: the researcher's perspective on thyroid testing in the Psittacine birds. Proc. Assoc. Avian Vet. Annu. Conf., p.89-94, 2009.

GREENACRE, C.B.; YOUNG, D.W., BEHREND, E.N. et al. Validation of a novel high-sensitivity radioimmunoassay procedure for measurement of total thyroxine concentration in psittacine birds and snakes. Am. J. Vet. Re.s, v.62, p.1750-1754, 2001.

GROSCOULAS, R.; LELOUP, J. The endocrine control of reproduction and moult in male and female emperor (Aptenodytes forsteri) and Adelie (Pygoscelis adeliae) penguins. Gen. Comp. Endocrinol, v.63, p.264-274, 1986.

JOHNSON, C.A. Reproductive manifestations of thyroid disease. Vet. Clin. N. Am. Small Anim. Pract., v.24, p.509-514, 1994.

LEMOS, GG.; CUNHA, I.C.N.; CONFORTI, V.A. et al. Concentração dos metabólitos de estradiol e progesterona fecais no cachorro-do-mato-vinagre (Speothosvenaticus) (Lund, 1842) pelos métodos de radioimunoensaio e quimioluminescência. Rev. Bras. Reprod. Anim., v.39, p.289-295, 2015. 
LOUKOPOULOS, P.; BAUTISTA, A.C.; PUSCHNER, B. et al. An outbreak of thyroid hyperplasia (goiter) with high mortality in budgerigars (Melopsittacus undulatus). J. Vet. Diagn. Invest., v.2, p.18-24, 2014.

MAYER, J.; DONNELLY, T.M. Clinical veterinary advisor birds and exotic pets. St Louis: Elsevier Saunders, 2013. 1061p.

MCNABB, F.M.A. Thyroids. In: WHITTOW, G.C. Sturkie's avian physiology. 5.ed. San Diego: Academic Press, 2000. p.461-471.

MERRYMAN, J.I.; BUCKLES, E.L. The avian thyroid gland. Part two: a review of function and pathophysiology. J. Avian Med. Surg., v12, p. 238242,1998

MULLER, K.; MULLER, E.; KLEIN, R. et al. Serum thyroxine concentrations in clinically healthy pet guinea pigs (Cavia porcellus). Vet. Clin. Pathol., v.38, p.507- 510, 2009.

NORRIS, D.O; CARR, J.A. Vertebrate endocrinology. 5.ed. San Diego: Academic Press, 2013. 580p.

OGLESBEE, B.L. Hypothyroidism in a scarlet macaw. J. Am. Vet. Med. Assoc., v.201, p.15991601, 1992.

OROSZ, S.E.; MONKS, D.; MATOS, R. Clinical endocrinology of the protein hormones. In: SPEER, B. (Ed.). Current therapy in avian medicine and surgery. Boston: Elsevier, 2016. p.379-382.

SCHMIDT, R.E.; REAVILL, D.R. The avian thyroid gland. Ve.t Clin. Exot. Anim., v.11, p.15-23, 2008.

SEGAL, J., TROEN, B.R., INGBAR, S.H. Influence of age and sex on the concentrations of thyroid hormone in serum in the rat. J. Endocrinol., v.93, p.177-181, 1982.
SHAMSIAN, A.A.; GHAZVINI, K.; SOKHTANLOO, M. et al. Which quantitative method in determination of the thyroid hormone levels is more consistent with the clinical symptom of the thyroid disorders? Comp. Clin. Pathol., v.25, p.101-106, 2016.

SILVERIN, B.; VIEBKE, P.A.; WESTIN, J. et al. Seasonal changes in body weight, fat depots and plasma levels of thyroxine and growth hormone in free-living Parus major and Parus montanus. Gen. Comp. Endocrinol., v.73, p.404-416, 1989.

SINGH, A.K.; JIANG, Y.; WHITE, T. et al. Validation of nonradioactive chemiluminescent immunoassay methods for the analysis of thyroxine and cortisol in blood samples obtained from dogs, cats, and horses. J. Vet. Diagn. Invest., v.9, p.261268, 1997.

STATISTICAL analysis systems. Versão 9.2. Cary: SAS Institute, 2009.

TEARE, J.A. Reference ranges for physiological values in captive wildlife. [Londres]: International Species Information System, 2002. CD-ROM.

VALLE, S.F.; ALLGAYER, M.C.; PEREIRA, A.R. et al. Parâmetros de bioquímica sérica de machos, fêmeas e filhotes de arara-canindé (Ara ararauna) saudáveis mantidas em cativeiro comercial. Ciênc. Rural, v.38, p.711-716, 2008.

VIEIRA, A.B.; CASTRO, M.C.; FREIRE, I.M.A. et al. Dosagem de tiroxina total (T4) sérica pelo método de quimioluminescência em gatos clinicamente sadios. Braz. J. Vet. Res. Anim. Sci., v.47, p.224-230, 2010.

WELCKER, J.; CHASTEL, O.; GABRIELSEN, G.W. et al. Thyroid hormones correlate with basal metabolic rate but not field metabolic rate in a wild bird species. Plos One, v.8, e56229, 2013. 